\title{
Artesunate and sorafenib: Combinatorial inhibition of liver cancer cell growth
}

\author{
HAO LI, KANGHE XU, GUANGZHE PIAN and SHU SUN \\ Department of Hepatopancreatobiliary Surgery, Affiliated Hospital of Yanbian University, Yanji, Jilin 133000, P.R. China
}

Received October 29, 2018; Accepted June 14, 2019

DOI: $10.3892 / 01.2019 .10810$

\begin{abstract}
An antimalarial medication, artesunate (Art), has exhibited promising anticancer effects with excellent tolerability in various types of cancer, suggesting that it has the potential to be used in combination with sorafenib (Sora) in hepatocellular carcinoma (HCC) treatment. To determine the potency of this combination, the present study attempted to quantitatively measure the dose-effect relationship of each drug alone and in combination in liver cancer cells in vitro using Calcusyn software. Cell growth inhibition was determined using the CyQUANT proliferation assay in two liver cancer cell lines, HepG2 and Huh7. Drug combination and reduction indices and isobologram plots were used to assess drug interactions. Cell apoptosis was evaluated by measurements of the proportion of cells in the sub $G_{0} / G_{1}$ phase of the cell cycle, and determination of protein expression levels of cleaved poly ADP ribose polymerase and caspase-9. Additionally, a cell migration assay was conducted using Essen ImageLock plates with an IncuCyte Zoom imaging system. The results of the present study revealed that the inhibitory effect of Sora on cell growth was synergistically enhanced by the combination with Art in HepG 2 and Huh7 cells. The combination index and dose reduction index were specific to each cell line. Furthermore, combination at a fixed ratio presented mutual enhancement with respect to apoptosis induction and suppression of in vitro liver cancer cell migration. Therefore, considering the low toxicity and well-defined clinical characteristics of Art, combination of Sora and Art may present an attractive therapeutic option in the development of clinical trials for HCC treatment.
\end{abstract}

Correspondence to: Dr Hao Li, Department of Hepatopancreatobiliary Surgery, Affiliated Hospital of Yanbian University, 1,327 Juzi Street, Yanji, Jilin 133000, P.R. China E-mail: lih@ybu.edu.cn

Key words: liver cancer cells, sorafenib, artesunate, synergistic effects

\section{Introduction}

Hepatocellular carcinoma (HCC) is one of the leading causes of morbidity and mortality worldwide. Despite substantial efforts and advances in drug discovery and clinical trials for advanced-stage HCC, there is a limited number of available effective treatments. Sorafenib (Sora), a multikinase inhibitor, exhibits significant effects on improvement of overall survival (OS), but it only delays tumor progression (1). To the best of our knowledge, first-line therapy beyond Sora or attempts to combine Sora with another targeted agent have not been successful, thus far (2).

Artesunate (Art) is a semisynthetic derivative of artemisinins and has been used worldwide over the past decade to combat severe malaria (3). Previous studies have demonstrated the anticancer effects of Art in various types of cancer, including leukemia, colorectal cancer, renal cell carcinoma, lung cancer and ovarian cancer (4-11). The primary mechanism of antitumor activity of Art is via reactive oxygen species generated by reactions between Art and iron that has accumulated in tumor cells $(6,8,11)$. Given its therapeutic significance, the pharmacokinetics of Art and its active metabolite dihydroartemisinin (DHA) have been intensively studied in clinical settings $(3,12)$. Art is rapidly absorbed and converted to its main active metabolite DHA, and generally cleared within an hour (12). This rapid clearing may contribute to its excellent tolerability and lack of adverse effects, even at high dose or rapid infusion. Accordingly, strategies combining Art with other drugs are being tested in vitro and in vivo to combat high systemic toxicity and chemoresistance, which limit the outcomes of cancer treatment (13-16).

Furthermore, Art has been demonstrated to decrease cell viability in a dose-dependent manner and increase caspase-3 activity in human and mouse liver cancer cell lines, HepG2 and BWTG3 (17). Additionally, it has been reported that Art may function as a potential inhibitor of STAT3 in HCC, and that Art modulates STAT3 targets (procaspase-3, B cell lymphoma 2 like 1 and survivin), leading to apoptosis in vitro (18). Notably, Art inhibits angiogenesis by directly downregulating the expression levels of vascular endothelial growth factor (VEGF) and its receptor (VEGFR) (17,19). Administration of Art reduces vascularization/tumor burden in xenograft mice, and when combined with Sora, these effects are further enhanced (17). This suggests that the combination of Sora and Art could be an effective treatment strategy for HCC. However, more reliable and standardized methods need to be employed in order to evaluate the potency of this drug combination prior to clinical trials. 
The present study attempted to quantitatively evaluate the type of drug interaction between Sora and Art by median-drug effect analysis using Calcusyn software (Chou-Talalay method). Drug combination and reduction indices and isobologram plots were applied to define drug interactivity in in vitro-cultured liver cancer cell lines, HepG2 and Huh7. Furthermore, the combinatorial effect of these two drugs on apoptosis induction and cell migration suppression was investigated for clinically achievable concentrations.

\section{Materials and methods}

Cell lines and reagents. The liver cancer cell lines, HepG2 and Huh7, were obtained from American Type Culture Collection, and were cultured in DMEM and RPMI medium (Thermo Fisher Scientific, Inc.), respectively. Both cell lines were supplemented with 10\% FBS (HyClone; GE Healthcare Life Sciences) and maintained in a humidified atmosphere containing $5 \% \mathrm{CO}_{2}$ at $37^{\circ} \mathrm{C}$. Cell line authentication was performed by short tandem repeat profiling and interspecies contamination test (Applied Biological Materials Inc.). Cryopreserved normal primary human hepatocytes (PHH) derived from a pool of 5 donors were purchased from Sekisui XenoTech, LLC. and thawed with OptiThaw Hepatocyte Media (Sekisui XenoTech, LLC.) according to the manufacturer's protocol. Cells were seeded in 96-well BioCoat ${ }^{\mathrm{TM}}$ collagen I cellware (BD Biosciences) in OptiPlate hepatocyte media (Sekisui XenoTech, LLC.) for $4 \mathrm{~h}$ until sufficient confluency was reached. The media was then replaced with OptiCulture hepatocyte media containing Penicillin/Streptomycin (Sekisui XenoTech, LLC.) and the cells were further incubated for $48 \mathrm{~h}$ for the hepatotoxicity assays. The multi-kinase inhibitor, Sora, was ordered from LC Laboratories and dissolved in dimethyl sulfoxide (DMSO). Art was purchased from TGI Chemicals, Ltd. and dissolved in $100 \%$ ethanol.

Antibodies. Rabbit anti-VEGF receptor 2 (D5B1; cat. no. 9698; 1:1,000), rabbit anti-cleaved PARP (D64E10; cat. no. 5625, 1:1,000), rabbit anti-cleaved caspase 9 (D8I9E; cat. no. 9505; 1:1,000) and rabbit anti-GAPDH (14C10; cat. no. 2118; 1:2,000) were all purchased from Cell Signaling Technology Inc.. HRP conjugated goat anti-rabbit secondary antibody (cat. no. sc-2004; 1:1,000) was purchased from Santa Cruz Biotechnology Inc.

Cell viability and drug combination assay. At $72 \mathrm{~h}$ prior to drug treatments, HepG2 and Huh7 cells $\left(5 \times 10^{3}\right.$ cells/well) were seeded in 96-well plates. Cells were treated with Sora, starting at 40 and $20 \mu \mathrm{M}$ for HepG2 and Huh7, respectively, and Art starting at 400 and 1,000 $\mu \mathrm{M}$ for HepG2 and Huh7, respectively, or the constant combination ratio of Sora to Art (1:10 in HepG2 and 1:50 in Huh7). To examine the hepatotoxicity of the combination of treatments, normal primary hepatocytes were treated in two constant combination ratios, 1:10 starting at 10 and $100 \mu \mathrm{M}$ for Sora and Art, respectively and at ratio of 1:50 starting at 20 and $1,000 \mu \mathrm{M}$ for Sora and Art, respectively. Cell viability was monitored using the CyQUANT Cell Proliferation Assay kit (Thermo Fisher Scientific, Inc.), according to the manufacturer's protocol. Cell morphology was observed under an inverted Nikon microscope and images were captured using a digital camera. Results from cells treated with individual drugs or the drug combination at a constant ratio (HepG2, Sora:Art, 1:10; Huh7, Sora:Art, 1:50) were processed, and drug combination and reduction indices and isobologram plots were calculated using the Calcusyn software v2.11 (Premier Biosoft International).

Western blot analysis. Protein extracts were prepared in $1 \%$ NP-40 lysis buffer (50 mM sodium fluoride, $1 \mathrm{mM}$ orthovanadate, $10 \mathrm{mM}$ iodoacetamide, $1 \mathrm{mM}$ ethylenediaminetetraacetic acid, $0.25 \%$ sodium deoxycholate, $1 \mathrm{mM}$ phenylmethylsulfonyl fluoride and protease inhibitor cocktail). Protein concentrations were determined by Pierce BCA protein assay kit (Thermo Fisher Scientific, Inc.). Cell protein lysates (50 $\mu \mathrm{g})$ were separated by $10 \%$ SDS-PAGE gels and then transferred onto PVDF membranes. The membranes were blocked with Tris-buffered saline containing 3\% BSA and $0.05 \%$ Tween-20 (TBST) for $30 \mathrm{~min}$, followed by incubation with aforementioned primary antibodies overnight at $4^{\circ} \mathrm{C}$ and aforementioned secondary antibody for $1 \mathrm{~h}$ at room temperature. A total of three 5-min washes with TBST were performed after incubation with the primary or secondary antibodies. The protein expression was detected using RapidStep ${ }^{\mathrm{TM}}$ Enhanced Chemiluminescence (Merck_KGaA). GAPDH was used as a reference protein.

Wound healing assay. HepG2 and Huh7 cells (20,000 cells/well) were seeded in triplicate in ImageLock 96-well plates (Essen Bioscience). Cells were treated with $1 \mathrm{mg} / \mathrm{ml}$ mytomycin $\mathrm{C}$ for $2 \mathrm{~h}$ in order to inhibit proliferation prior to wound scratching. Confluent cell layers were scratched using the Essen Bioscience Wound Maker in order to generate wounds that were 700-800 $\mu \mathrm{m}$ wide. Cells were washed twice with PBS and allowed to grow in $10 \%$ FBS growth medium with vehicle (DMSO or $100 \%$ ethanol), $2.5 \mu \mathrm{M}$ Sora, $25 \mu \mathrm{M}$ (HepG2)/125 $\mu \mathrm{M}$ (Huh7) Art or Sora + Art. Images were captured at $6 \mathrm{~h}$ intervals for $36 \mathrm{~h}$ using the IncuCyte ZOOM imaging system with time-lapse bright field microscopy (Essen Bioscience). Relative wound density was calculated based on the ratio of cell density in the wound/cell density outside the wound.

Flow cytometry. Liver cancer cell lines were plated into 6-well plates at a density of 300,000 cells/well and treated with vehicle, $2.5 \mu \mathrm{M}$ Sora, $25 \mu \mathrm{M}$ (HepG2)/125 $\mu \mathrm{M}$ (Huh7) Art or Sora + Art for $72 \mathrm{~h}$. Cell cycle analysis with propidium iodide (PI) staining was performed according to a standard protocol. Briefly, drug-treated cells were harvested in cell suspensions in PBS and fixed in a final concentration of $70 \%$ ethanol on ice for $30 \mathrm{~min}$, followed by incubation with the PI/Triton X-100 staining solution $(50 \mathrm{mg} / \mathrm{ml}$ PI, $0.05 \%$ Triton X-100 and $100 \mathrm{mg} / \mathrm{ml}$ RNase A) at $37^{\circ} \mathrm{C}$ for $1 \mathrm{~h}$. Data was acquired by collecting the area and width on a linear scale for the DNA channel in addition to forward scatter and side scatter. Relative DNA contents were analyzed using the FACSCanto II flow cytometer and BD FACSDiva software v5.0.3 (Becton, Dickinson and Company). Apoptotic cells were indicated by the percentage of $\operatorname{subG}_{0} / \mathrm{G}_{1}$ phase.

Statistical analysis. All values are presented as the means \pm standard deviation of at least three independent experiments. Statistical analyses were performed to analyse 
differences in growth inhibition and apoptosis using one-way ANOVA followed by Tukey's post hoc analysis. The wound scratch assays were analysed using Friedman's test followed by the Dunn's post hoc test using GraphPad Prism v6.0.1 software (GraphPad Software, Inc.). P<0.05 (two-tailed) was considered to indicate a statistically significant difference.

\section{Results}

Determination of dose-response curves and half-maximal inhibitory concentration $\left(I C_{50}\right)$ values in liver cancer cell lines. To determine accurate $\mathrm{IC}_{50}$ values of Sora and Art used in the present study, dose-response curves were initially generated for these drugs using the CyQUANT cell proliferation assay. This assay is a cell metabolic activity-independent and sensitive method, which is used to directly measure DNA content to quantify cells. The dose response curves of Sora in HepG2 and Huh7 cells were similar to those reported previously, with $\mathrm{IC}_{50}$ values of 5.93-8.51 (mean 7.10) $\mu \mathrm{M}$ in HepG2 and 7.11-17.11 (mean 11.03) $\mu \mathrm{M}$ in Huh7 cells (Fig. 1; Table I) $(20,21)$. It has been reported that the maximum drug concentration of Art in human plasma is 3,260 $(1,020-164,000) \mathrm{ng} / \mathrm{ml}[8.48(2.65-427.08) \mu \mathrm{M}]$ and the terminal elimination half-life is $0.25(0.1-1.8) \mathrm{h}(22)$. To obtain clinically relevant concentrations, the inhibitory dose response of Art was tested for $72 \mathrm{~h}$ in the two cell lines at a starting concentration of $1,000 \mu \mathrm{M}$. This revealed that the $\mathrm{IC}_{50}$ values of Art ranged between 63.28 and 99.85 (mean 79.49) $\mu \mathrm{M}$ in HepG2, and 344.70-1,099 (mean 615.40) $\mu \mathrm{M}$ in Huh7 cells (Fig. 1; Table I). The results indicated that HepG2 and Huh7 cells exhibited low sensitivity to Art treatment, compared with other types of cancer cell lines, including leukemia or colon cancer cell lines, which have been reported to be responsive in vitro to Art at $1.11 \pm 0.56$ and $2.13 \pm 0.74 \mu \mathrm{M}$, respectively (4). However, the benefits of the drug combination include mutual enhancement of therapeutic effects, prevention of drug resistance and reduction of dose used per single treatment.

Combinatorial inhibition of Sora and Art of liver cancer cell growth. To leverage the synergistic inhibitory effects of Sora and Art, a constant combination ratio of Sora to Art (1:10 in HepG2 and 1:50 in Huh7) was used, based on $\mathrm{IC}_{50}$ values of the individual drugs in liver cancer cell lines. The results demonstrated that the combination of Sora and Art significantly augmented cell growth inhibition compared with single drug treatments, the starting concentrations at which this effect became significant were $2.5 \mu \mathrm{M}$ for Sora combined with Art at $25 \mu \mathrm{M}$ for HepG2 and $2.5 \mu \mathrm{M}$ Sora with $125 \mu \mathrm{M}$ Art for Huh7 (Fig. 2). In addition, $\mathrm{PHH}$ were tested for the potential hepatotoxic effects associated with this drug combination. Due to the limitations of the utility of this model, the hepatotoxicity evaluation here is focused on cell viability. The cells were treated with the drug combination ratios of Sora to Art, 1:10 and 1:50, with Art starting at $100 \mu \mathrm{M}$ and $1,000 \mu \mathrm{M}$, respectively. The former ration did not present hepatotoxicity in human normal primary hepatocytes, whereas with the latter ratio, Art treatment alone at higher concentrations $(>250 \mu \mathrm{M}$ ), significantly reduced the cell viability by $\sim 40 \%$ (the combination ratio at 1:50). Notably, no significant difference was observed between Art alone and the combination treatments, suggesting that the drug combination in PHH does not show augmented hepatotoxicity beyond the single drug treatments.
Table I. Cytotoxic effects of sorafenib and artesunate in liver cancer cell lines.

Half-maximal inhibitory concentration, $\mu \mathrm{M}$ (95\% confidence interval)

\begin{tabular}{lcc}
\cline { 2 - 3 } Drug & HepG2 cells & Huh7 cells \\
\hline Sorafenib & $7.10(5.93-8.51)$ & $11.03(7.11-17.11)$ \\
Artesunate & $79.49(63.28-99.85)$ & $615.40(344.70-1099.00)$
\end{tabular}

Synergistic effects of Sora and Art on suppression of liver cancer cell growth. To further determine the types of drug interactions, the data were analyzed by median-drug effect analysis (Calcusyn) to determine antagonism [combination index $(\mathrm{CI})>1$ ], additivity $(\mathrm{CI}=1)$ and synergism $(\mathrm{CI}<1)$. The combinatorial effects of Sora and Art in HepG2 and Huh7 cells as identified by the CI, isobologram and dose reduction index (DRI) at three dose-effect levels of cell growth inhibition $\left(\mathrm{IC}_{50}, \mathrm{IC}_{75}\right.$ or $\mathrm{IC}_{90}$ ) are summarized in Table II. The combinations exhibited strong synergy in HepG2 cells (CI range, 0.11-0.22) or moderate to strong synergy in Huh7 cells (CI range, 0.75-0.43), which was also indicated by the isobolograms (Fig. 3; Table II). The isobolograms indicate the nature of the drug interaction at constant ratios in each liver cancer cell line. They present drug combination effects at the $\operatorname{ED}_{50}(50 \%$ effective dose), $\mathrm{ED}_{75}\left(75 \%\right.$ effective dose), and $\mathrm{ED}_{90}$ (90\% effective dose) and the data points below the line, on the line or above the line suggest synergistic, additive or antagonistic effects, respectively (Fig. 3). Although there are differences between HepG2 and Huh7 cells in response to this drug combination which showed opposing trends in the CIs and DRIs (Table II), the CIs clearly indicated strong synergism in HepG2 and moderate synergism in Huh7 cells. Furthermore, the DRIs exhibited considerable dose reduction for Sora and Art as a result of synergism. When applied at the indicated constant ratio (Table II), the $\mathrm{IC}_{50}$ of Sora was decreased 14.69-fold (HepG2) or 3.04-fold (Huh7). For Art, it was reduced 21.86-fold (HepG2) or 2.34-fold (Huh7). The dose reduction levels were specific to each cell line.

Additionally, a fixed constant ratio of drug combination (Sora:Art, 1:5) was tested in HepG2 and Huh7 cells for $48 \mathrm{~h}$ (Fig. S2). Art treatment at concentrations $<100 \mu \mathrm{M}$ demonstrated moderate effects on growth inhibition within $48 \mathrm{~h}$ (17-40\%) for the two cell lines, while it exhibited synergistic effects with Sora on suppression of cell growth in HepG2 cells as indicated by CI (Fig. S2A). By contrast, concurrent treatment of Art and Sora in Huh7 cells for $48 \mathrm{~h}$ did not comply with the Calcusyn mathematical model since the median-effect curves in this cell line exhibited a negative slope due to its poor response to Art treatment ( $<100 \mu \mathrm{M}$; Fig. S2B). However, this was overcome by $24 \mathrm{~h}$ pre-treatment with Art, followed by the combination treatment (Fig. S2B), which suggested that sequential treatment of Art followed by Sora may improve the overall outcome of this drug combination in liver cancer treatment. 

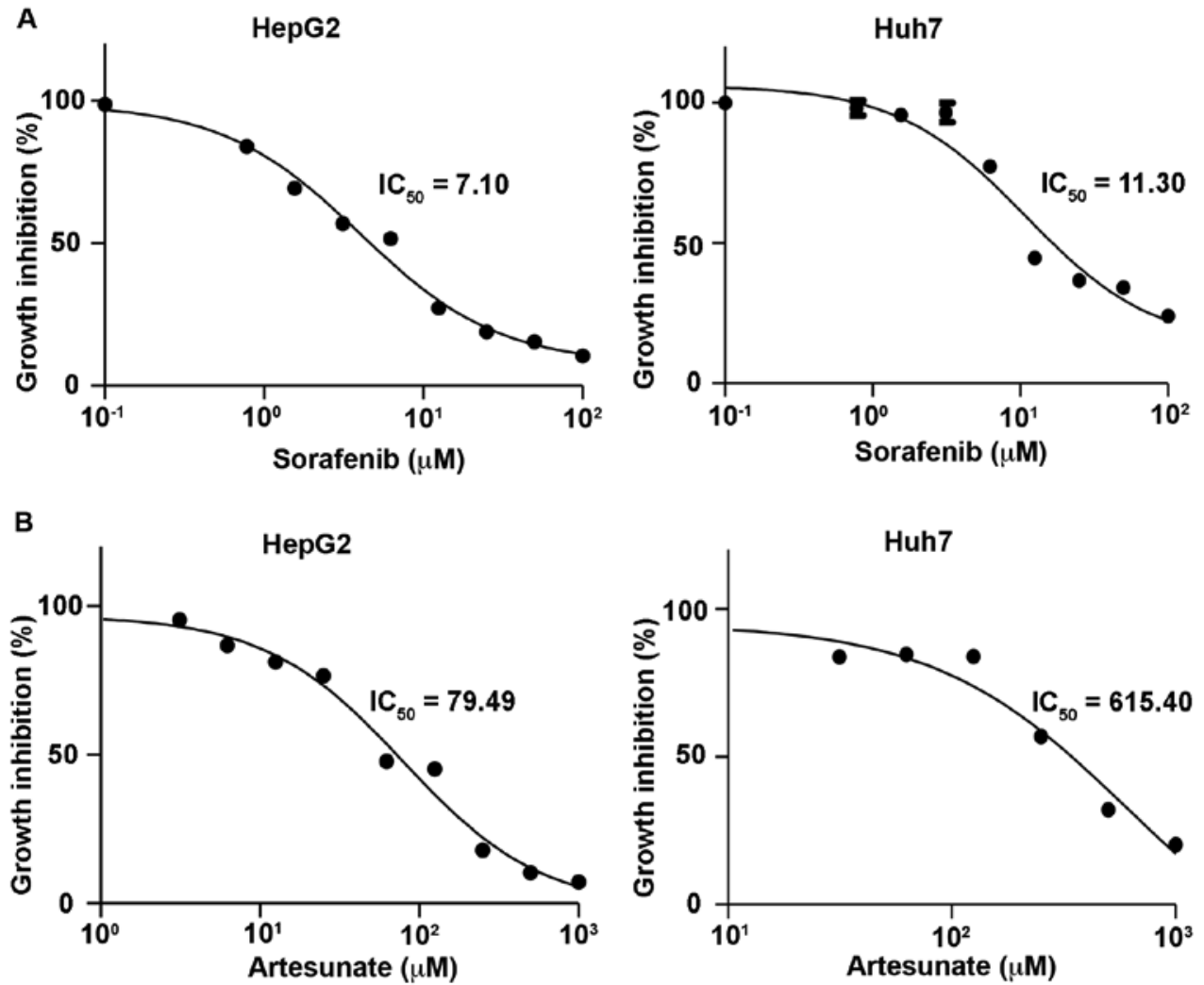

Figure 1. Dose-response curves and $\mathrm{IC}_{50}$ values for sorafenib and artesunate in liver cancer cells. HepG2 and Huh7 cells were treated with (A) sorafenib and (B) artesunate at different concentrations for $72 \mathrm{~h}$, followed by a CyQUANT proliferation assay. $\mathrm{IC}_{50}$, half-maximal inhibitory concentration.
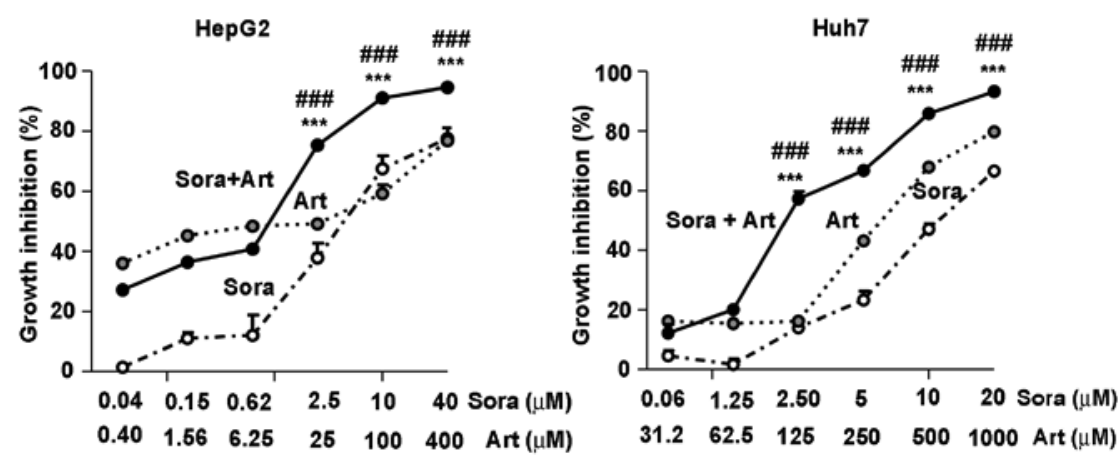

Figure 2. Combinatorial inhibitory effect of Sora and Art on cell growth in liver cancer cells in vitro. HepG2 and Huh7 cells were treated with Sora, Art, or Sora + Art at a constant ratio of Sora:Art, 1:10 (HepG2 cells) or 1:50 (Huh7 cells) for $72 \mathrm{~h}$ at different concentrations, as indicated. Cell growth inhibition was determined by a CyQUANT cell proliferation assay. Data are presented as the mean + standard deviation. ${ }^{* * *} \mathrm{P}<0.001$ vs. Sora. ${ }^{\# \#} \mathrm{P}<0.001 \mathrm{vs}$. Art. Sora, sorafenib; Art, artesunate.

Combination of Sora and Art significantly increases cell apoptosis. It has been previously reported that Art exerts anticancer properties in a variety of tumor types via the activation of mitochondrial apoptosis (17). Art also has been revealed to reduce tumor vessel formation via downregulation of VEGF and VEGFR protein expression levels $(12,14)$. To further assess the biological effects of the combination of Sora and Art, concentrations (Sora:Art, 2.5:25 $\mu \mathrm{M}$ for HepG2; 2.5:125 $\mu \mathrm{M}$ for Huh7) at which the combination started exhibiting noticeable enhancement of growth inhibition at $72 \mathrm{~h}$ compared with the single drug treatments were selected (Fig. 2). This significantly reduced cell numbers, which was partially caused by cell apoptosis induction as indicated by the percentage of cells in the subG $0 / G_{1}$ phases of the cell cycle (Fig. 4A and B). Consistent with these results, western blot analysis revealed that the initiator and the final substrate of the caspase cascade, cleaved caspase- 9 and cleaved poly ADP ribose polymerase expression levels were significantly increased following combination treatment. Sora and Art reduced VEGFR2 protein expression, and this effect was enhanced by drug combination in HepG2 and Huh7 cells (Fig. 4C).

Combination of Sora and Art significantly reduces cell migration. Subsequently, the combined effect of the drugs on cell migration was determined using an in vitro wound healing assay (Fig. 5). Cell migration was kinetically monitored in 
Table II. Calcusyn output of median-effect analysis of Sora and Art combination in liver cancer cell lines.

\begin{tabular}{|c|c|c|c|c|c|c|c|c|c|c|}
\hline \multirow[b]{2}{*}{ Cell line } & \multirow[b]{2}{*}{ Drugs } & \multicolumn{3}{|c|}{ Parameters } & \multicolumn{3}{|c|}{ CI value } & \multicolumn{3}{|c|}{ DRI value } \\
\hline & & $\operatorname{Dm}(\mu \mathrm{M})$ & $\mathrm{m}$ & $\mathrm{r}$ & $\mathrm{IC}_{50}$ & $\mathrm{IC}_{75}$ & $\mathrm{IC}_{90}$ & $\mathrm{IC}_{50}$ & $\mathrm{IC}_{75}$ & $\mathrm{IC}_{90}$ \\
\hline \multirow[t]{3}{*}{ HepG2 } & Sora & 5.48 & 0.76 & 0.98 & & & & 14.69 & 10.01 & 6.81 \\
\hline & Art & 81.5 & 0.69 & 0.93 & & & & 21.86 & 17.48 & 13.98 \\
\hline & Sora + Art (1:10) & $0.37+3.73$ & 0.6 & 0.97 & 0.11 & 0.16 & 0.22 & & & \\
\hline \multirow[t]{3}{*}{ Huh7 } & Sora & 16.44 & 1.33 & 0.91 & & & & 3.04 & 4.57 & 6.46 \\
\hline & Art & 631.93 & 1.62 & 0.87 & & & & 2.34 & 2.96 & 3.61 \\
\hline & Sora $+\operatorname{Art}(1: 50)$ & $5.39+270.05$ & 2.29 & 0.98 & 0.75 & 0.56 & 0.43 & & & \\
\hline
\end{tabular}

Dose-effect relationships were calculated by median-effect analysis using Calcusyn. $\mathrm{m}=1, \mathrm{~m}>1$ and $\mathrm{m}<1$ signify hyperbolic, sigmoidal and flat sigmoidal shapes, respectively. $r$ indicates conformity of results. $\mathrm{CI}<1, \mathrm{CI}=1$ or $\mathrm{CI}>1$, indicate synergism, additive or antagonism, respectively. DRI was measured by comparing the doses when used as a single treatment or in combination. Sora, sorafenib; Art, artesunate; CI, combination index; DRI, dose reduction index; Dm, median-effect dose that inhibits cell growth by $50 \%$; r, linear correlation coefficient; IC $_{50}$, half-maximal inhibitory concentration; $\mathrm{IC}_{75}, 75 \%$ inhibitory concentration; $\mathrm{IC}_{90}, 90 \%$ inhibitory concentration; $\mathrm{m}$, shape of curve.
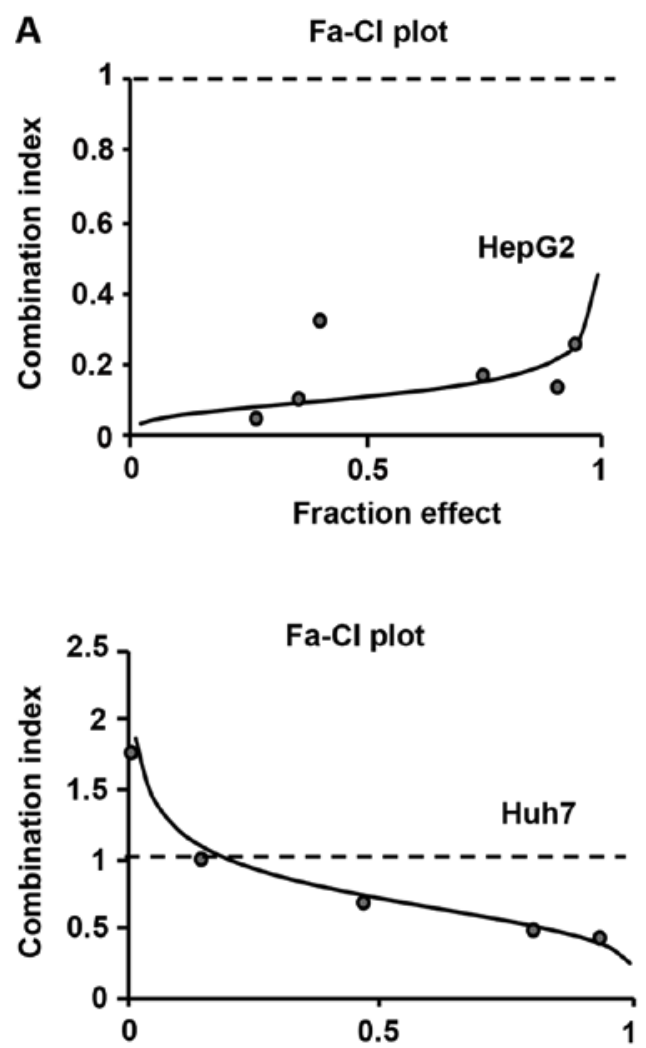

Fraction effect
B
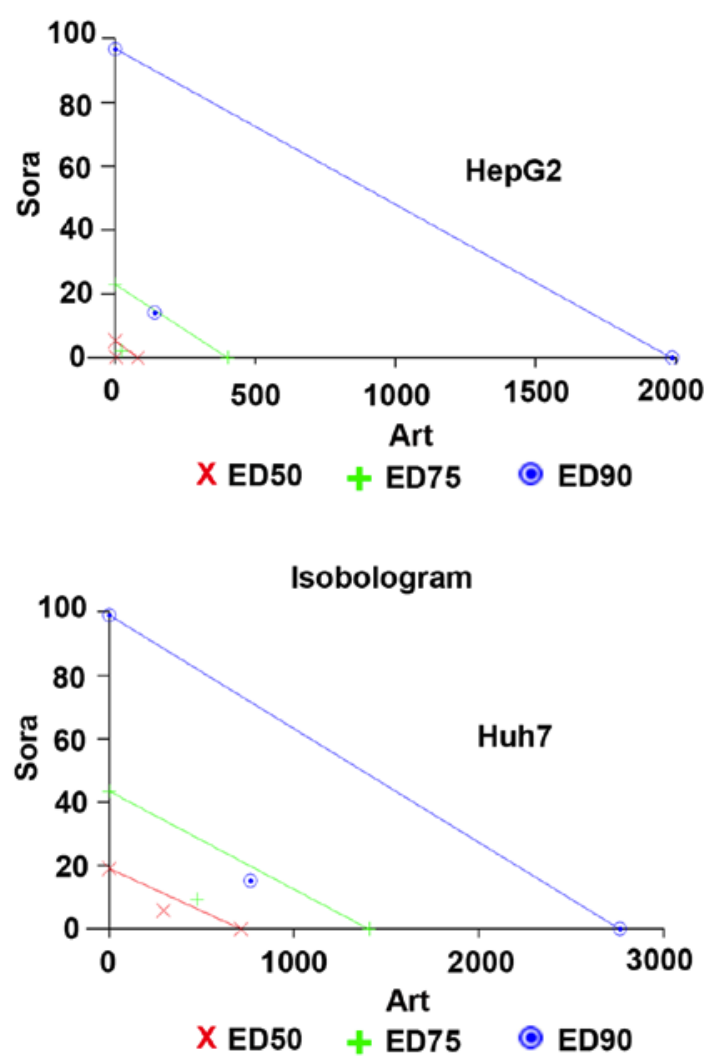

Figure 3. Dose-effect relationship of Sora and Art combination in liver cancer cell lines. (A) Fa-CI plots were obtained from the median-effect analysis (Calcusyn). Solid lines show computer-simulated Fa-CI plots. Circles represent experimental data points. $\mathrm{CI}<1, \mathrm{CI}=1$ and $\mathrm{CI}>1$ indicate synergism, additive effects and antagonism, respectively. (B) Isobolograms indicate the nature of the drug interaction at constant ratios in each liver cancer cell line. Respective drug combination at the $\mathrm{ED}_{50}, \mathrm{ED}_{75}$, and $\mathrm{ED}_{90}$ effect levels, data points below the line = synergistic, on the line $=$ additive and above the line $=$ antagonistic effects. The degree of synergism in this drug combination is reflected by the distance of the data point from its respective line (same color). Fa, fraction affected; CI, combination index; Sora, sorafenib; Art, artesunate; $\mathrm{ED}_{50}$, median effective dose to inhibit $50 \%$ of cells; $\mathrm{ED}_{75}$, median effective dose to inhibit $75 \%$ of cells; $\mathrm{ED}_{90}$, median effective dose to inhibit $90 \%$ of cells.

Essen ImageLock plates using the IncuCyte Zoom imaging system. Cell growth was controlled by mitomycin C treatment. For the selected drug combination ratio (Sora:Art, 2.5:25 $\mu \mathrm{M}$ for HepG2 and 2.5:125 $\mu \mathrm{M}$ for Huh7), no significant apoptotic effects were observed at $24 \mathrm{~h}$ (data not shown). However, the results revealed a substantial inhibition of migration in the combination-treated cells compared with vehicle, Sora or Art treated cells (Fig. 5A and B). 

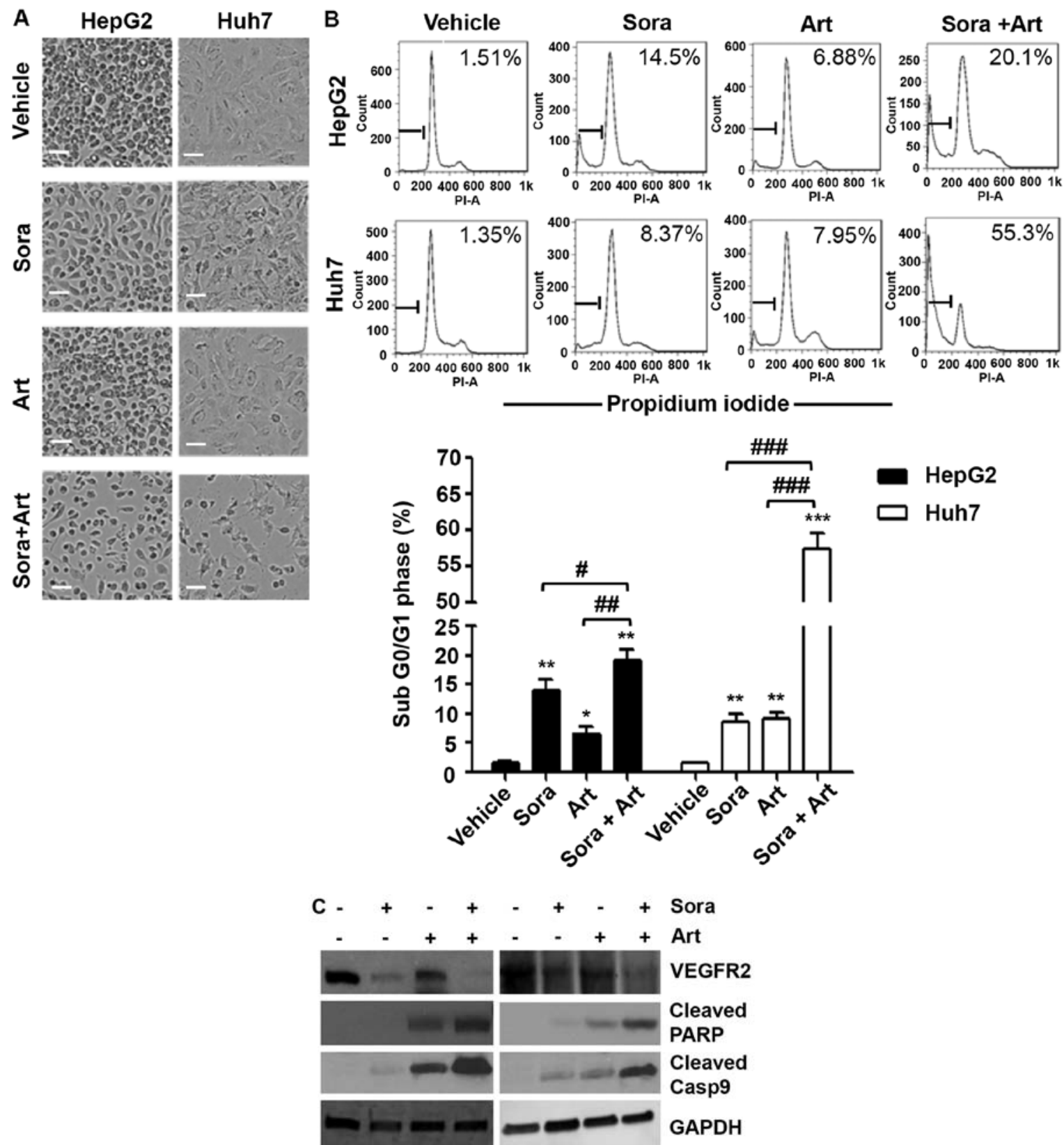

Figure 4. Combination of Sora and Art markedly increases apoptosis. (A) Morphological alterations of liver cancer cells treated with Sora, Art or Sora + Art. HepG2 and Huh7 cells were treated with $2.5 \mu \mathrm{M}$ Sora, $25 \mu \mathrm{M}$ (HepG2) or $125 \mu \mathrm{M}$ (Huh7) Art, or Sora + Art for 72 hrs, followed by phase-contrast microscopy. Scale bar, $100 \mu \mathrm{m}$. (B) Effects of Sora and Art on the induction of apoptosis in HepG2 and Huh7 cells. Cells were stained with PI and DNA contents were examined by flow cytometry. Apoptotic cells were gated in the subG $\mathrm{G}_{0} \mathrm{G}_{1}$ phase. (C) Western blot analysis of the VEGFR2 (Sora target) and apoptosis-associated proteins, cleavage and activation of initiator Casp9, and PARP proteolysis (effector; caspase-3 substrate). GAPDH was used as a loading control. Data are presented as the mean + standard deviation. ${ }^{*} \mathrm{P}<0.05,{ }^{* *} \mathrm{P}<0.01,{ }^{* * *} \mathrm{P}<0.001$ vs. vehicle control; ${ }^{\# P} \mathrm{P}<0.05,{ }^{\# \#} \mathrm{P}<0.01,{ }^{\# \# \#} \mathrm{P}<0.001$. Sora, sorafenib; Art, artesunate; $\mathrm{PI}$, propidium iodide; VEGFR2, vascular endothelial growth factor receptor 2; PARP, poly ADP ribose polymerase; Casp9, caspase-9.

\section{Discussion}

In the SHARP trial (2008), Sora treatment was associated with a modest improvement in survival (2.8 months) compared with placebo treatment; however, the treatment was commonly associated with adverse effects, resulting in discontinuation of the drug in certain cases (1). Since then, there have been a number of trials investigating the combination therapy of Sora with various interventions, including VEGF-targeted monoclonal antibody, bevacizumab (Avastin ${ }^{\circledR}$; Genentech) $(2,23)$. The overall assessment of the majority of studies is that they do not support the combination of two chemotherapeutic agents for the treatment of unresectable HCC (23-25). Recently, another multikinase inhibitor, regorafenib, first appeared to improve survival rates in patients who progressed on Sora, with an increased median OS over 24 months across the two lines of therapy with Sora as a first-line and regorafenib as a second-line treatment $(24,25)$. However, due to common adverse events, patients intolerant to Sora were excluded from the study (24). Therefore, further investigation is required to determine the benefits or lack of benefits of combination therapy. Similarly, identification of a synergistic partner drug would provide an opportunity for dose-reduction; and therefore, increase the therapeutic window. 
A

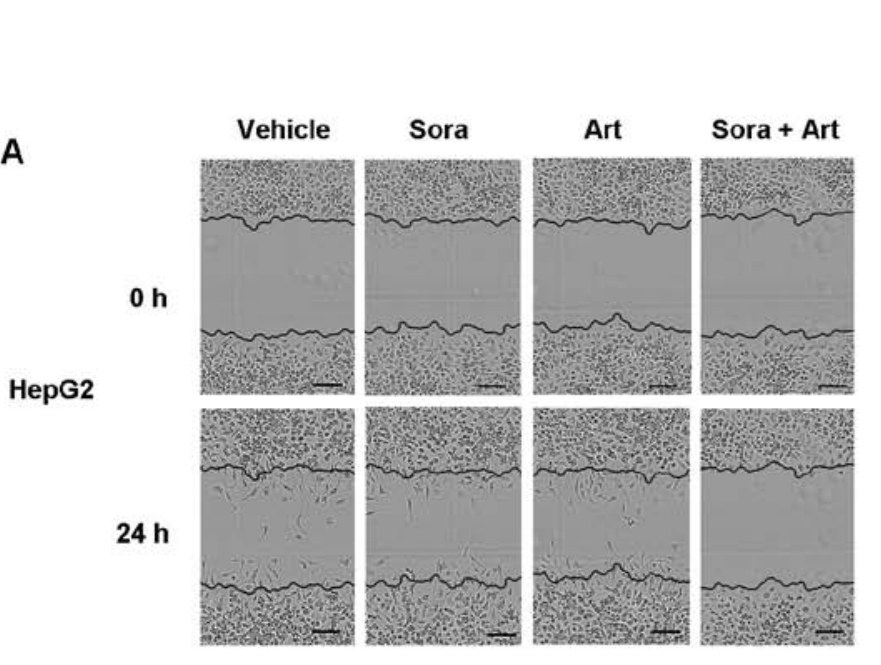

B

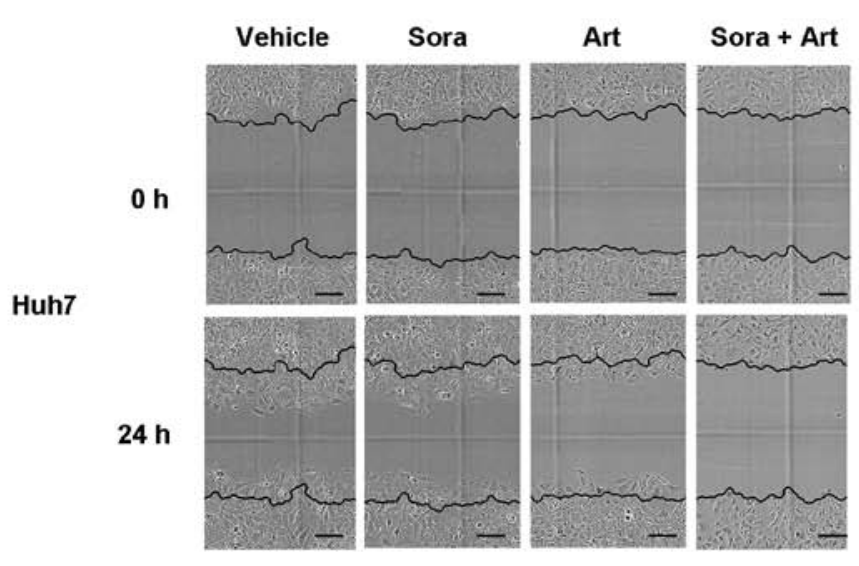

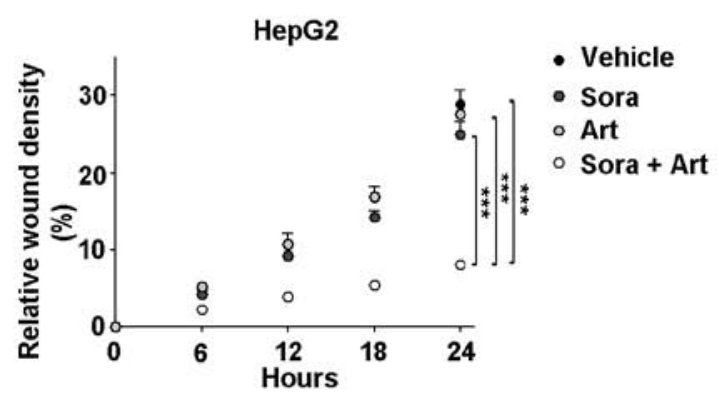

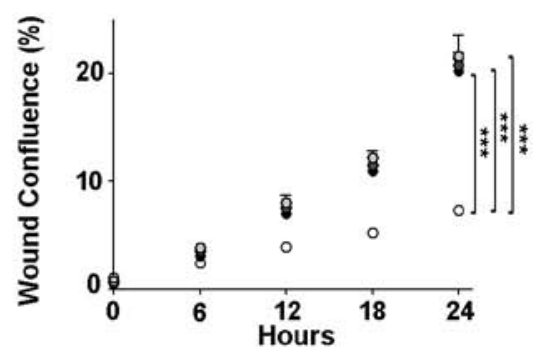

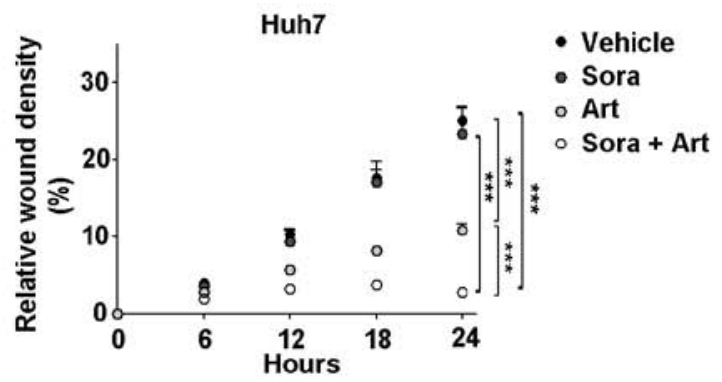

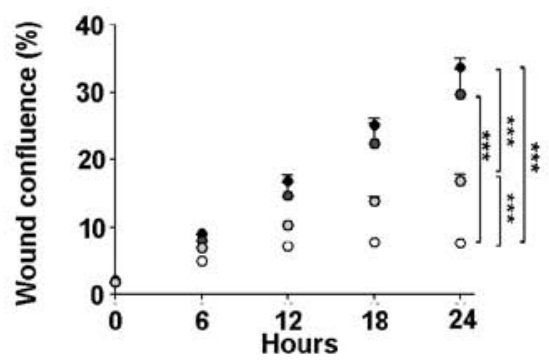

Figure 5. Combination of Sora and Art enhances the suppression of cell migration. HepG2 and Huh7 cells were seeded into Essen ImageLock 96-well plates and treated with mytomycin $\mathrm{C}$ for $2 \mathrm{~h}$ prior to wound scratching. Cells were treated with vehicle (dimethyl sulfoxide), Sora, Art or Sora + Art. Kinetic cell migration was recorded by the IncuCyte ZOOM imaging system at $6 \mathrm{~h}$ intervals for $36 \mathrm{~h}$. (A) Top, representative images of wound healing assay of HepG2 cells treated with indicated drugs in wound healing assay at 0 and $36 \mathrm{~h}$. The initial scratch wound is shown in blue lines. Bottom, relative wound density of HepG2 was measured to quantify cell migration. Scale bar, $300 \mu \mathrm{m}$. (B) Relative wound density of Huh7 at $36 \mathrm{~h}$. Data are presented as the mean + standard deviation. ${ }^{* * *} \mathrm{P}<0.001$. Sora, sorafenib; Art, artesunate.

Drug repurposing of Art in cancer therapy has been proposed in numerous studies (4-11). Synergistic effects of Art and other chemotherapy drugs have been reported in a number of types of cancer $(15,16,26-28)$. Therefore, its combination with Sora has been investigated in HCC in in vitro and in vivo (17). However, the lack of evidence from reliable and standardised methods defining the in vitro synergistic potential of this drug combination remains a major drawback for its practical use. It is often unclear whether it has greater or lesser effects in combination with other drugs compared with the simple additive effect expected from the combination of the effects of each drug individually. This is a great limitation in providing valuable insights for developing drug combination in cancer therapeutics. In the present study, an effort was made to measure the dose-effect relationship of Sora or Art alone or in combination, and to quantitatively determine whether this combination yielded a synergistic effect in liver cancer cells.

Median-drug effect analysis (Calcusyn) was used to define drug interactivity by generating the CI, isobologram and DRI in an objective manner. The drug combination ratio reported in the present study was determined based on the $\mathrm{IC}_{50}$ values of each drug, and the same ratio was applied to additional functional studies. The results of the present study indicated that combination at the fixed ratio was associated with strong to moderate synergistic growth inhibition in HepG2 and Huh7 cells. The DRI of Sora ranged between 14.69- and 3.04-fold and that of Art ranged between 21.86- and 2.34-fold at the $\mathrm{IC}_{50}$ level. The synergistic effects 
included apoptosis induction, cell migration inhibition and anti-angiogenesis activity. Notably, the combination treatment reduced VEGFR2 protein expression more than Sora or Art alone, indicating these two drugs cooperatively exert anti-angiogenesis roles. Additionally, another drug combination ratio of Sora:Art (1:5) was applied in HepG2 and Huh7 cells, and sequential combination treatment in Huh7 cells that were not responding well in the first setting was assessed. It was revealed that the drug combinatorial effect of Art and Sora was synergistic. The benefit of this particular drug combination in HCC is not only due to the characteristics of the drugs, but also dependent on the dose ratio and scheduling of treatments. Furthermore, it would be reasonable to consider what dose ratio or scheduling of treatments may optimize the synergy. Ideally, these two factors should be extensively optimized in preclinical studies prior to proceeding to a clinical setting in humans.

In conclusion, the present study provided methodological evidence to facilitate the development of the drug combination of Sora and Art in HCC treatment. This could be a potential treatment option for patients with $\mathrm{HCC}$ and may harness the overall therapeutic efficacy of Sora with Art, an affordable and well-characterized medication. The combination ratio and concurrent/sequential dosing schedule requires further investigation in clinical trials.

\section{Acknowledgements}

The authors would like to thank Dr Na Li (Vancouver Prostate Centre, Vancouver, BC, Canada) for their advice regarding experimental design and data analysis.

\section{Funding}

The present study was supported by research funding from Yanbian University (Jilin, China) for junior investigators (grant no. 20120010) and the Natural Science Foundation of Jilin Province, China (grant no. 201115239).

\section{Availability of data and materials}

The datasets used and/or analyzed in the present study are available from the corresponding author on reasonable request.

\section{Authors' contributions}

HL contributed to the overall experimental design, performed the experiments, and analyzed the data for the cell growth assay and cell migration assay. KX and GP contributed to western blot analysis and flow cytometry. SS contributed to the experimental design, data interpretation and preparation of the manuscript.

\section{Ethics approval and consent to participate}

Not applicable.

\section{Patient consent for publication}

Not applicable.

\section{Competing interests}

The authors declare that they have no competing interests.

\section{References}

1. Llovet JM, Ricci S, Mazzaferro V, Hilgard P, Gane E, Blanc JF, de Oliveira AC, Santoro A, Raoul JL, Forner A, et al: Sorafenib in advanced hepatocellular carcinoma. N Engl J Med 359: 378-390, 2008

2. Sun W and Cabrera R: Systemic treatment of patients with advanced, unresectable hepatocellular carcinoma: Emergence of therapies. J Gastrointest Cancer 49: 107-115, 2018.

3. Dondorp A, Nosten F, Stepniewska K, Day N, White N and South East Asian Quinine Artesunate Malaria (SEAQUAMAT) group: Artesunate versus quinine for treatment of severe falciparum malaria: A randomised trial. Lancet 366: 717-725, 2005.

4. Efferth T, Dunstan H, Sauerbrey A, Miyachi H and Chitambar CR: The anti-malarial artesunate is also active against cancer. Int J Oncol 18: 767-773, 2001.

5. Crespo-Ortiz MP and Wei MQ: Antitumor activity of artemisinin and its derivatives: From a well-known antimalarial agent to a potential anticancer drug. J Biomed Biotechnol 2012: 247597, 2012.

6. Kumar B, Kalvala A, Chu S, Rosen S, Forman SJ, Marcucci G, Chen CC and Pullarkat V: Antileukemic activity and cellular effects of the antimalarial agent artesunate in acute myeloid leukemia. Leuk Res 59: 124-135, 2017.

7. Krishna S, Ganapathi S, Ster IC, Saeed ME, Cowan M, Finlayson C, Kovacsevics H, Jansen H, Kremsner PG, Efferth T and Kumar D: A randomised, double blind, placebo-controlled pilot study of oral artesunate therapy for colorectal cancer. EBioMedicine 2: 82-90, 2015.

8. Chauhan AK, Min KJ and Kwon TK: RIP1-dependent reactive oxygen species production executes artesunate-induced cell death in renal carcinoma Caki cells. Mol Cell Biochem 435: 15-24, 2017.

9. Tong Y, Liu Y, Zheng H, Zheng L, Liu W, Wu J, Ou R, Zhang G, Li F, Hu M, et al: Artemisinin and its derivatives can significantly inhibit lung tumorigenesis and tumor metastasis through Wnt/beta-catenin signaling. Oncotarget 7: 31413-31428, 2016.

10. Zhang CZ, Zhang H, Yun J, Chen GG and Lai PB: Dihydroartemisinin exhibits antitumor activity toward hepatocellular carcinoma in vitro and in vivo. Biochem Pharmacol 83: 1278-1289, 2012.

11. Greenshields AL, Shepherd TG and Hoskin DW: Contribution of reactive oxygen species to ovarian cancer cell growth arrest and killing by the anti-malarial drug artesunate. Mol Carcinog 56: 75-93, 2017.

12. Morris CA, Duparc S, Borghini-Fuhrer I, Jung D, Shin CS and Fleckenstein L: Review of the clinical pharmacokinetics of artesunate and its active metabolite dihydroartemisinin following intravenous, intramuscular, oral or rectal administration. Malar J 10: 263, 2011 .

13. Efferth T, Giaisi M, Merling A, Krammer PH and Li-Weber M: Artesunate induces ROS-mediated apoptosis in doxorubicin-resistant T leukemia cells. PLoS One 2: e693, 2007.

14. Wang B, Hou D, Liu Q, Wu T, Guo H, Zhang X, Zou Y, Liu Z, Liu J, Wei J, et al: Artesunate sensitizes ovarian cancer cells to cisplatin by downregulating RAD51. Cancer Biol Ther 16: 1548-1556, 2015.

15. Efferth T: Cancer combination therapy of the sesquiterpenoid artesunate and the selective EGFR-tyrosine kinase inhibitor erlotinib. Phytomedicine 37: 58-61, 2017.

16. Nunes JJ, Pandey SK, Yadav A, Goel S and Ateeq B: Targeting NF-kappa B signaling by artesunate restores sensitivity of castrate-resistant prostate cancer cells to antiandrogens. Neoplasia 19: 333-345, 2017.

17. Vandewynckel YP, Laukens D, Geerts A, Vanhove C, Descamps B, Colle I, Devisscher L, Bogaerts E, Paridaens A, Verhelst X, et al: Therapeutic effects of artesunate in hepatocellular carcinoma: Repurposing an ancient antimalarial agent. Eur J Gastroenterol Hepatol 26: 861-870, 2014.

18. Ilamathi M, Santhosh S and Sivaramakrishnan V: Artesunate as an anti-cancer agent targets stat-3 and favorably suppresses hepatocellular carcinoma. Curr Top Med Chem 16: 2453-2463, 2016. 
19. Chen HH, Zhou HJ, Wu GD and Lou XE: Inhibitory effects of artesunate on angiogenesis and on expressions of vascular endothelial growth factor and VEGF receptor KDR/flk-1. Pharmacology 71: 1-9, 2004.

20. Cervello M, Bachvarov D, Lampiasi N, Cusimano A, Azzolina A, McCubrey JA and Montalto G: Molecular mechanisms of sorafenib action in liver cancer cells. Cell Cycle 11: 2843-2855, 2012.

21. Liu J, Liu Y, Meng L, Ji B and Yang D: Synergistic antitumor effect of sorafenib in combination with ATM inhibitor in hepatocellular carcinoma cells. Int J Med Sci 14: 523-529, 2017.

22. Byakika-Kibwika P, Lamorde M, Mayito J, Nabukeera L, Mayanja-Kizza H, Katabira E, Hanpithakpong W, Obua C, Pakker N, Lindegardh N, et al: Pharmacokinetics and pharmacodynamics of intravenous artesunate during severe malaria treatment in Ugandan adults. Malar J 11: 132, 2012.

23. Hubbard JM, Mahoney MR, Loui WS, Roberts LR, Smyrk TC, Gatalica Z, Borad M, Kumar S and Alberts SR: Phase I/II randomized trial of sorafenib and bevacizumab as first-line therapy in patients with locally advanced or metastatic hepatocellular carcinoma: North central cancer treatment group trial N0745 (Alliance). Target Oncol 12: 201-209, 2017.

24. Bruix J, Qin S, Merle P, Granito A, Huang YH, Bodoky G, Pracht M, Yokosuka O, Rosmorduc O, Breder V, et al: Regorafenib for patients with hepatocellular carcinoma who progressed on sorafenib treatment (RESORCE): A randomised, double-blind, placebo-controlled, phase 3 trial. Lancet 389: 56-66, 2017.
25. Finn RS, Merle P, Granito A, Huang YH, Bodoky G, Pracht M, Yokosuka O, Rosmorduc O, Gerolami R, Caparello C, et al: Outcomes of sequential treatment with sorafenib followed by regorafenib for HCC: Additional analyses from the phase III RESORCE trial. J Hepatol 69: 353-358, 2018.

26. Tran BN, Nguyen HT, Kim JO, Yong CS and Nguyen CN: Developing combination of artesunate with paclitaxel loaded into poly-d,l-lactic-co-glycolic acid nanoparticle for systemic delivery to exhibit synergic chemotherapeutic response. Drug Dev Ind Pharm 43: 1952-1962, 2017.

27. Goswami U, Kandimalla R, Kalita S, Chattopadhyay A and Ghosh SS: Polyethylene glycol-encapsulated histone deacetylase inhibitor drug-composite nanoparticles for combination therapy with artesunate. ACS Omega 3: 11504-11516, 2018.

28. Efferth T: Cancer combination therapies with artemisinin-type drugs. Biochem Pharmacol 139: 56-70, 2017.

This work is licensed under a Creative Commons Attribution-NonCommercial-NoDerivatives 4.0 International (CC BY-NC-ND 4.0) License. 